\title{
The Effect of Exercise on Urinary Gamma-Glutamyltransferase and Protein Levels in Elite Female Karate Athletes
}

\author{
Nader Shavandi ${ }^{1}$, PhD; Abolfazl Samiei*1, MSc; Reza Afshar ${ }^{2}$, MD; \\ Abbas Saremi ${ }^{1}$, PhD; Rahman Sheikhhoseini ${ }^{1}$, MSc
}

Authors' Affiliation:

1. Department of Sports Physiology, Arak University, Arak, Iran

2. Department of Nephrology, Shahed University, Tehran, Iran

* Corresponding Author;

Address: Physical Education and Sport Sciences Department, Humanity

Faculty, Arak University, Shariati Sq,

Arak, Iran

E-mail: abolfazl.samiee@gmail.com

Received: May 24, 2011

Accepted: Sep 22, 2011

Key Words: Athletes; Creatinine; Gamma-Glutamyltransferase;

Martial Arts; Renal Function; Protein

\begin{abstract}
Purpose: Post exercise proteinuria and increased urinary Gamma-Glutamyl transferase (GGT) levels can be indicative of exercise-induced renal damage. The aim of this investigation is to study the effect of one session of intensive training on renal damage markers and compare their values to those 6 hours after training.

Methods: In this cross-sectional study with pre- and post-test design, 10 elite volunteer female athletes were selected and participated in one training session ( 2 hours). Urine samples were collected before training, one hour after training, and 6 hours after training. Urinary protein (Pr), creatinine (Cr), and GGT values were measured through laboratory methods and then $\mathrm{Pr} / \mathrm{Cr}$ and $\mathrm{GGT} / \mathrm{Cr}$ ratios were computed.
\end{abstract}

Results: There were significant differences between values of protein, GGT and Creatinine in the three sampling phases $(P<0.05)$. However, no significant differences were observed between values for $\mathrm{GGT} / \mathrm{Cr}$ and $\mathrm{Pr} / \mathrm{Cr}$ ratio. There were significant differences between the mean values of Creatinine, protein and GGT within pre-exercise and 1 hour post-exercise and within 1 hour postexercise and 6 hours post-exercise $(P<0.05)$.

Conclusion: It seems that a session of karate training does not result in renal damage and athletes can continue training after 6 hours.

Asian Journal of Sports Medicine, Volume 3 (Number 1), Mar 2012, Pages: 41-46

\section{INTRODUCTION}

In recent years, the number of athletes joining martial arts has increased significantly ${ }^{[1]}$. Karate is a globally widespread martial art, which means "Empty Hand" in Japanese $(\mathrm{Kara}=$ Empty, $\mathrm{Te}=$ Hand $)$. This reveals the philosophy of karate in which athletes do Punches, kicks and blocks without the use of weapons ${ }^{[2]}$. Although the positive effects of exercise on human health has been known for many years, professional sports can still cause complications in renal ${ }^{[3]}$, cardiovascular ${ }^{[4]}$, and muscular systems ${ }^{[5]}$. By reducing the renal circulation rate and the glomerular filtration rate, exercise can result in proteinuria ${ }^{[6]}$.
Actually, exercise results in protein excretion to urine, especially protein that is available in blood plasma. This state is called "exercise proteinuria" which occurs during vigorous exercise or immediately after that, and it depends on the intensity and duration of training. High intensity/short duration training can increase glomerular and tubular proteinuria, while moderate intensity/long duration training increases proteinuria, especially due to the decrease of tubular protein reabsorption ${ }^{[7]}$.

Some experimental studies showed that GammaGlutamyl transferase (GGT) is a membranous enzyme that has a critical role in glutathione homeostasis by initiating extracellular glutathione breakdown and it 
acts as a critical cellular antioxidant defense ${ }^{[8]}$. This enzyme is primarily located on the luminal surface of cellular membranes of proximal tubule epithelial cells, thus its highest activity can be found in kidneys ${ }^{[9]}$.

Increasing the enzymes excretion to urine can be due to renal damage and the type of urinary enzymes can determine the primary site of renal damage, too, because every part of anephron has a specific enzyme quality ${ }^{[10]}$. Several studies have been done investigating the effects of exercise on urinary protein and creatinine, yet the number of studies on urinary

\section{METHODS AND SUBJECTS}

The subjects were the karate athletes of Arak team, one of the sport clubs of the main league of Iran (Autumn 2010). Research methods were approved by Medical University of Arak's Ethics Committee (NO: 89-84-2). The exclusion criteria were the presence of any history of serious/chronic diseases or any history of renal diseases and the risk of a drug-induced nephrotoxicity. Ten karate athletes were selected ranging in age from 18 to 26 years. The test was done in one day by giving a training program with the following schedule: general and karate-specific warm-up for 30 minutes, main stage of workout (i.e. Yuri Tsuki, Mawashi Gery and Tsuki,) for 2 minutes each, in 2 sets and 2 minutes rest; Meet's Tsuki and Mawashi Gery for 30 seconds each, in 3 sets and 1 minute rest; rapid Tsuki , Mawashi Gery and Kusacherica Tsuki,10 seconds for each movement, in 3 sets and 1 minute rest, and 15 minutes of recovery.
The total duration of the training was 120 minutes and totally included 3 sampling times, first, before the training started; 1 hour post-training, and 6 hours posttraining the karate athletes gave clean-catch urine samples in sterile containers. Urinary GGT levels were measured by GTSL method and urinary creatinine levels were measured using the JAFFE reaction and no adjustments were done for daily creatinine variations. In order to minimize errors due to changes in urine flow, GGT levels were given as ratios toward urinary creatinine levels and urinary protein levels were determined according to the Bradford method using bovine serum albumin as the standard. Variance analysis using general linear model-repeated measures test was conducted to assess the significance of differences among the three measurements. Also, dependent $\mathrm{t}$-test was run to determine the difference between the two sample pairs using commercially available statistics software (SPSS 18). $P$-value $<0.05$ was considered statistically significant.

\section{RESULTS}

Demographic characteristics of the female karate athletes are as shown in table 1 and their urinary findings are shown in Tables 2 and 3. Pre-training GGT $[\mathrm{u} / \mathrm{l}]$ levels increased to a higher amount after 1 hour post training, and decreased after 6 hours posttraining to an amount similar to the initial. The only statistically significant increase in GGT levels was that of the 1 hour post-training $(\mathrm{P}<0.05)$. GGT/Cr $(\mathrm{u} / \mathrm{g}$ creatinine) levels increased both 1 and 6 hours after post-training compared to the pre training, but they were not considered significantly different $(\mathrm{P}>0.05)$.

Table 1: Demographic characteristics of female karate athletes

\begin{tabular}{|lcc|}
\hline variable & Mean (Standard Deviation) & Range \\
\hline Age(years) & $20.37(2.12)$ & $18-25$ \\
Weight(Kg) & $56.25(6.53)$ & $46-68$ \\
Height(Cm) & $166.90(4.12)$ & $161-173$ \\
BMI(Kg/m ${ }^{2}$ ) & $20.20(1.97)$ & $16.49-22.76$ \\
Sports history(years) & $9.35(2.90)$ & $6-15$
\end{tabular}


Table 2: Comparing urinary factors of female karate athletes in the three times of sampling with Repeated Measures Test

$\begin{array}{lcccc}\text { Variable } & \text { Before training } & \mathbf{1} \text { hour after training } & \mathbf{6} \text { hours after training } & \boldsymbol{P} \text {. value } \\ \text { Protein(mg/ml) } & 3.17(1.14) & 13.23(10.81) & 3.95(1.49) & 0.02 \\ \text { Creatinine(mg/dl) } & 100.50(56.79) & 168.00(53.29) & 64.70(25.70) & <0.001 \\ \text { GGT(U/l) } & 57.40(31.51) & 137.60(49.01) & 58.60(13.84) & <0.001 \\ \text { Protein/creatinine } & 0.04(0.03) & 0.08(0.07) & 0.07(0.04) & 0.2 \\ \text { GGT(u/g creatinine) } & 0.77(0.86) & 0.92(0.47) & 1.04(0.40) & 0.7\end{array}$

creatinine levels increased to a higher value after 1 hour post-training, which was found to be the only significant increase of creatinine levels from pretraining to post-training $(P<0.05)$. It also decreased after the 6 hour post-training in comparison with the pre-training values. Proteinuria level was considered to be higher 1 hour post-training and similar to the pretraining after the 6 hours post-training. The only significant difference was the increase of proteinuria level 1 hour after post-training compared to pretraining $(P<0.05)$. It was shown that proteinuria/ creatinine ratio 1 hour post-training and 6 hours posttraining increases, but they were not found to be significant $(P<0.05)$.

\section{DISCUSSION}

The results of this analysis show that one session of intensive karate training increases the urinary level of renal damage markers in female karate athletes. But these exercise-induced changes decrease to initial values over 6 hours post-training. The findings show significant differences between the average protein values sampled at 3 different times. However, the average protein values increased 1 hour post-training, and decreased 6 hours after the training session which reveals no significant differences compared to the initial values (See Tables 2 and 3).

Ayca et al (2008) observed no significant differences between pre, post, and 24 hours after training urinary protein values of gymnasts which is in contrast to the findings of our research ${ }^{[12]}$. Another study by Ayca et al (2006) reported significant differences in the levels of urinary protein changes after one training session in male volleyball athletes but had no follow-up period [11].

Various studies showed that urinary protein values increase after one training session. The amount of this increase depends more on the intensity of the exercise than the duration ${ }^{[13]}$. Generally, due to the size and electrical charge of protein molecules, they are filtered through the glomerular capillary membrane from which about 90 percent is absorbed via renal tubular cells. In general, proteinuria is due to increased filtration, reduced protein re-absorption, or both. Various studies have shown that in our case, both mechanisms take part; however, the increased

Table 3: The difference and significant levels between the means related to sample pairs, Wilcoxon Test, Dependent t-Test

\begin{tabular}{|c|c|c|c|c|c|c|c|c|c|}
\hline \multirow[t]{2}{*}{ Variable } & \multicolumn{3}{|c|}{ Before and 1 hour after training } & \multicolumn{3}{|c|}{1 hour and 6 hour after training } & \multicolumn{3}{|c|}{ Before and $\mathbf{6}$ hour after training } \\
\hline & $\begin{array}{l}\text { Mean of } \\
\text { changes }\end{array}$ & $95 \% \mathrm{CI}$ & $\begin{array}{c}P . \\
\text { Value }\end{array}$ & $\begin{array}{l}\text { Mean of } \\
\text { changes }\end{array}$ & $95 \% \mathrm{CI}$ & $\begin{array}{c}P . \\
\text { Value }\end{array}$ & $\begin{array}{l}\text { Mean of } \\
\text { changes }\end{array}$ & $95 \%$ CI & $\begin{array}{c}P . \\
\text { Value }\end{array}$ \\
\hline Protein (mg/ml) & $-10.06(3.28)$ & $-17.49-2.63$ & 0.01 & $9.30(3.47)$ & $1.42 \_17.13$ & 0.02 & $-0.78(0.70)$ & $-2.36 \_0.80$ & 0.3 \\
\hline Creatinine (mg/dl) & $-67.50(21.07)$ & $-115.17-19.83$ & 0.01 & $103.30(19.21)$ & $59.85 \_146.75$ & $<0.001$ & $35.80(21.22)$ & $-12.21 \_83.81$ & 0.1 \\
\hline GGT (U/l) & $-80.20(16.98)$ & $-118.60 \_-41.80$ & 0.001 & $79.00(16.43)$ & $41.82 \_116.18$ & 0.001 & $-1.20(12.26)$ & $-28.92 \_26.52$ & 0.9 \\
\hline Protein/creatinine & $-0.04(0.02)$ & $-0.09 \_0.01$ & 0.1 & $0.01(0.02)$ & $-0.04 \_0.06$ & 0.1 & $-0.029(0.02)$ & $-0.07 \_0.01$ & 0.6 \\
\hline GGT/Cr & $-0.016(0.36)$ & $-0.99 \_0.68$ & 0.1 & $-0.11(0.23)$ & $-0.62 \_0.40$ & 0.7 & $-0.27(0.34)$ & $-1.03 \_0.50$ & 0.07 \\
\hline
\end{tabular}

CI: Confidence Interval 
glomerular capillary permeability mechanism is more effective than protein re-absorption. Generally, the anionic property in the glomerular membrane confronts the crossing of the protein molecules with negative charge. During exercise, the albumin permeability of the membrane increases due to reduction in the negative charge of the

glomerular membrane. Previously, it was believed that severe renal vasoconstriction due to exercise causes proteinuria, but recent studies have shown that renal prostaglandins can be a cause for exercise-induced proteinuria too, since using prostaglandin inhibitor drugs before training dramatically decreases the value of exercise-induced proteinuria showing no change in renal hemodynamics ${ }^{[14]}$.

The results of this research also revealed that average creatinine levels increase one hour post exercise compared to pre-exercise and then decrease 6 hours post exercise which all happen to be statistically significant changes. Although insignificant differences were observed between the average data pre and 6 hours post-exercise which mean that the creatinine level returns to its initial level 6 hours post-exercise $(\mathrm{P}<0.05)$.

Although several studies show an increase of urinary creatinine level post exercise ${ }^{[10]}$, many researchers have reported insignificant increase of creatinine ${ }^{[11,12]}$. The differences observed when comparing the results of these research studies may be due to the intensity, the duration of training, or the age of volunteers. Creatinine is a substance formed by decomposition of creatine in muscles, filtered through kidneys easily before entering urine. The urinary creatinine levels can be a renal filtration marker. The more the renal filtration, the higher the creatinine urinary levels are [15].

Since renal the excretion value of protein and creatinine is related to the value of water excretion, these values vary throughout the day ${ }^{[16]}$. Provided that the glomerular filtration level remains constant, the urinary excretion of protein and creatinine throughout the day will remain adequately constant. So it seems that the protein/creatinine ratio undergoes less continuous changes than the independently introduced values of creatinine and protein ${ }^{[17]}$. The evaluation of the protein/creatinine ratio obtained in this study showed no significant differences in ratio values of all 3 sampling times. The results are similar to the results of Ayca et al (2006) ${ }^{[11]}$ and Ayca et al (2008) ${ }^{[12]}$ but in contrast to those of Scarpa et al (2007) ${ }^{[18]}$.

Although the average values of GGT increased from $57.40 \pm 31.51$ pre exercise to $137.60 \pm 49.01,1$ hour post exercise but decreased again to $58.60 \pm 13.84,6$ hours post exercise $(\mathrm{P}<0.05)$, that difference was not significantly different from the pre-exercise value $(P>0.05)$. The average values of GGT/creatinine ratio, in order to minimize urinary flow rate, were calculated in all 3 times of sampling which showed no significant differences either $(\mathrm{P}>0.05)$.

Ayca et al (2006) ${ }^{[11]}$ found no significant differences between the GGT values pre and post exercise in female and male volleyball players but it was significant for spikers. Due to performing more explosive and jumping movements, it seems that one exercise session causes more renal tubule damage in spikers than other volleyball players. The findings of studies by Ayca et al (2008) ${ }^{[12]}$ and Scarpa et al (2007) ${ }^{[18]}$ showed significant changes in

this enzyme's values post exercice.

The highest activity of GGT is found in proximal tubule epithelial cells, so this enzyme can be a marker for some renal diseases ${ }^{[19]}$. The amount of excretion of some renal enzymes, such as leucine aminopeptidase, alkaline phosphatase, and GGT increases in renal damage. It seems that the increase in GGT excretion can be due to damage of proximal renal tubules ${ }^{[20]}$. According to existing evidence, the increased urinary GGT level may be due to two mechanisms: The first mechanism is either complete or partial ischemia of kidneys that prevents oxygenation of tubular epithelium and leads to reduction in cellular energy values and discharge of ATP reserves that trigger biochemical events responsible for fatal damages and finally cell death ${ }^{[21]}$. The second one may be due to myoglobinoria of rhabdomyolysis. In this case, the myoglobin entering kidneys (which is toxic to kidneys) causes renal cells damage and release of GGT ${ }^{[22]}$. Our study faced some limitations in the number of volunteers and the sampling time periods. It was better if more subjects attend the study and sampling takes place several times post exercise, renal function would be determined more precisely. It is recommended that 
in future studies the values for urinary creatinine be measured at the same exact times on days other than the exercise day in order to assess the reproducibility of the test results. Nevertheless, the changes can be due to exercise since the pre-exercise values of each individual were compared to the post-exercise values of the same individual.

\section{CONCLUSION}

Results of this study show that although absolute values of creatinine, protein, GGT, and protein/creatinine ratio increase after one training session, the GGT/creatinine ratio changes are not significant. Finally, each of the abovementioned values decrease to its initial value 6 hours later. Therefore, it seems that one session of karate training cannot lead to renal damage and athletes can exercise 6 hours later.

\section{ACKNOWLEDGMENTS}

This study was funded by the Research vice-President of Arak University; we gratefully acknowledge them and thank the athletes and Markazi laboratory's personnel for the invaluable help they offered.

\section{Conflict of interests: None}

\section{REFERENCES}

1. Pieter W, Lufting R. Injuries at the 1991 taekwondo world championships. J Sports Traumatol Related Res 1994:16;49-57.

2. Halabchi F, Ziaee V, Lotfian S. Injury profile in women Shotokan Karate Championships in Iran (2004-2005). J Sports Sci Med 2007; $6: 52-57$

3. Neumayr G, Pfister R, Hoertnagl H, et al. Renal Function and Plasma Volume Following Ultramarathon Cycling. Int J Sports Med $2005 ; 26: 2-8$

4. Konig D, Schumacher YO, Heinrich L, et al. Myocardial Stress after Competitive Exercise in Professional Road Cyclists. Med Sci Sports Exerc 2003;35:1679-83

5. Clarkson P, Kearns A, Rouzier P, et al. Serum Creatine Kinase Levels and Renal Function Measures in Exertional Muscle Damage. Med Sci Sports Exerc 2006;38:623-7.

6. Abarbanel J, Benet AE, Lask D, Kimche D. Sports hematuria. J Urol 1990;143:887-90.

7. Tipton CM. Exercise physiology: people and ideas. New York: Oxford University Press. 2003; P:466.

8. Karp DR, Shimooku K, Lipsky PE. Expression of gamma-glutamyl transpeptidase protects Ramos B cells from oxidation-induced cell death. J Biol Chem 2001;276:3798-804.

9. Tate SS, Meister A. Gamma-glutamyl transpeptidase from kidney. Methods Enzymol 1985;113:400-19.

10. Turgut G, Kaptanoğlu B, Turgut S, et al. Influence of acute exercise on urinary protein, creatinine, insulin-like growth factor (IGF-1) and IGF binding protein-3 concentration in children. Tohoku J Exp Med 2003;201:165-70.

11. Ayca B, Sener A, Apikoglu Rabus S, Oba R. The effect of exercise on urinary gamma-glutamyl transferase and protein leves of volleyball players. J Sports Med Physical Fitness 2006;46:623-7.

12. Ayça B, Agopyan A, Sener A, et al. Evaluation of gamma-glutamyl transferase changing in urine related to the training load in the rythmic gymnasts competitors aged 7-10. Biol Sport 2008;25:233-44.

13. Bellinghieri G, Savica V, Santoro D. Renal alterations during exercise. J Ren Nutr 2008;18:158-64.

14. American College of Sports Medicine (Unanimous). ACSM's advanced exercise physiology. Baltimore: Lippincott Williams \& Wilkins. 2006; Pp:526.

15. Delanghe J, De Slypere JP, De Buyzere M, et al. Normal reference values for creatine, creatinine, and carnitine are lower in vegetarians. Clin. Chem 1989;35:1802-3.

16. Moore RR, Hirate-Dulas CA, Kasiske BL. Use of urine specific gravity to improve screening for albuminuria. Kidney Int 1997;52: 240-3. 
17. Price CP, Newall RG, Boyd JC. Use of Protein:Creatinine Ratio Measurements on Random Urine Samples for Prediction of Significant Proteinuria: A Systematic Review. Clin Chem 2005:51:1577-86.

18. Scarpa P, Di Fabio V, Ramirez C, et al. Proteinuria, GGT Index and Fractional Clearance of Electrolytes in Exercising Athletic Horses. Vet Res Commu 2007;31:339-42.

19. Nemesanszky E, Lott JA. Gamma-glutamyl transferase and its iso enzymes: progress and problems. Clin Chem 1985;31:797-803.

20. Vanderlinde RE. Urinary enzyme measurements in the diagnosis of renal disorders. Ann Clin Lab Sci 1981;11:189-201.

21. Henry JB. Henry's Clinical Diagnosis and Management by Laboratory Methods. 20 ${ }^{\text {th }}$ Ed, 2001, Transleted by: Asgari M, Setareshenas R, Shayanfar N. Tehran: Iran's University of Medical Sciences Publishing. 2002.

22. Clarkson PM, Kearns AK, Rouzier P, et al. Serum creatine kinase levels and renal function measures in evectional muscle damage. Med Sci Sports Exerc 2006;38:623-7. 